DENDRITIC CELLS

\section{pDCs rely on type I IFNs}

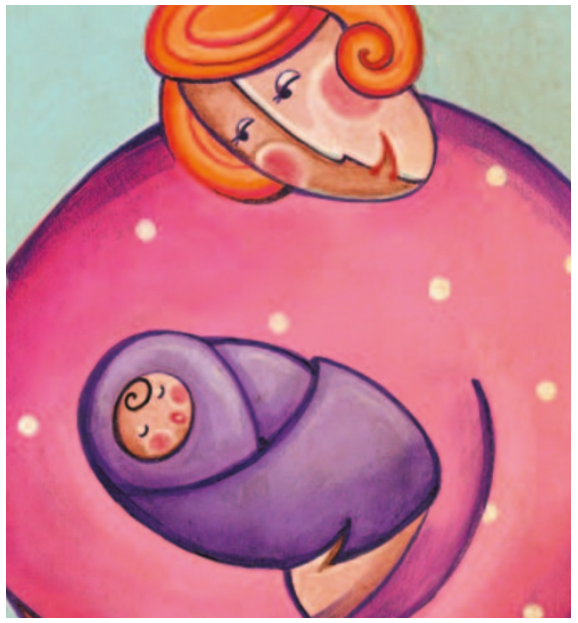

Distinct dendritic cell (DC) subsets express different patterns of Toll-like receptors (TLRs), and this has been proposed to determine the type of immune response that is induced by microorganisms. However, a recent study published in The Journal of Experimental Medicine now indicates that both plasmacytoid DCs (pDCs) and conventional DCs (cDCs) are between the two DC subsets. activated in vivo by ligands for TLR4, TLR7 and TLR9 but that the type of response and its dependence on type I interferons (IFNs) differs

In this study, Asselin-Paturel et al. set out to investigate which DC subset produces type I IFNs - cytokines (such as IFN- $\alpha$ and IFN- $\beta$ ) that have an important role in the antiviral immune response and that have been suggested to promote DC maturation - after in vivo stimulation with TLR ligands. As expected, administration of a TLR7 or TLR9 ligand (resiquimod or CpG-containing oligodeoxynucleotides (ODNs), respectively), but not a TLR4 ligand (lipopolysaccharide, LPS), to wildtype mice induced production of type I IFNs by pDCs but not by $\mathrm{cDCs}$. The production of type I IFNs in response to CpG ODNs, but not resiquimod, was dependent on expression of the type I IFN receptor (IFNAR), indicating that in vivo production of type I IFNs in response to distinct TLR ligands is differentially regulated.

In contrast to type I IFN production, LPS, resiquimod and $\mathrm{CpG}$ ODNs each induced in vivo upregulation of CD40 and CD86 expression by pDCs and cDCs. In vivo activation of pDCs by each of these ligands was dependent on IFNAR expression; however, only resiquimodand $\mathrm{CpG}-\mathrm{ODN}$-mediated activation of cDCs was IFNAR dependent. By contrast, $\mathrm{CpG}$ ODNs and resiquimod, but not LPS, induced IFNAR-dependent migration of pDCs to the marginal zone of the spleen and the outer T-cell area, where they formed clusters, whereas all three ligands induced the migration of cDCs to the T-cell area of the spleen, in an IFNAR-independent manner. Further analysis indicated that pDCs from mice treated with CpG ODNs showed reduced chemotaxis towards the CXC-chemokine receptor 3 (CXCR3) ligand CXC-chemokine ligand 9 (CXCL9) and increased chemotaxis towards the CC-chemokine receptor 7 (CCR7) ligands CC-chemokine ligand 19 (CCL19) and CCL21. Interestingly, in vivo induction of CXCL9 and CXCL10 expression in response to CpG ODNs was IFNAR dependent, leading the authors to suggest that this could partly explain the dependence of CpG-ODN-mediated pDC migration on type I IFNs.

Karen Honey

(2) References and links

ORIGINAL RESEARCH PAPER Asselin-Paturel, C. et al.

Type I interferon dependence of plasmacytoid dendritic cell activation and migration. J. Exp. Med. 28 Mar 2005 (doi:10.1084/jem20041930)

\title{
Inflammatory combinations
}

Induction of an inflammatory response in the cervix following infection with human papillomavirus (HPV) might lead to an increased risk of cervical cancer - this is the conclusion of a new study by Mary Carrington and colleagues.

Natural killer (NK) cells are an important component of the innate immune response to viruses and tumours, and their activation depends on the balance of signals from inhibitory and activating receptors, such as the killer-cell immunoglobulin-like receptors (KIRs). KIRs engage specific HLA class I proteins at the surface of target cells, and the numerous possible KIR and HLA haplotypes mean that there is huge scope for variation in HLA-KIR interactions. Carrington et al. show that certain combinations of HLA class I and KIR alleles are associated with a higher risk of developing cervical cancer.

The authors examined the frequencies of HLA class I alleles among women with and without cervical cancer. They found that some HLA allelic groups that are known to encode high-affinity ligands for inhibitory KIRs - HLA-Cw group 2 and $H L A-B w 4$ alleles - are associated with reduced risk of the disease. They also found that some activating KIRs - such as KIR3DS1 are expressed at a higher frequency in individuals who have cervical cancer than in those who do not. Furthermore, when the authors examined the effects of carrying KIR3DS1 together with $H L A-C w$ group 2 and $H L A-B w 4$ alleles, they found a gradient of susceptibility to cervical cancer.

Carrington and colleagues point out that lesions in women with oncogenic HPV infections are often associated with concomitant inflammation of the cervix, and they suggest that NK-cell activation might promote the development of cancer by contributing to local inflammation. However, although inflammation has been associated

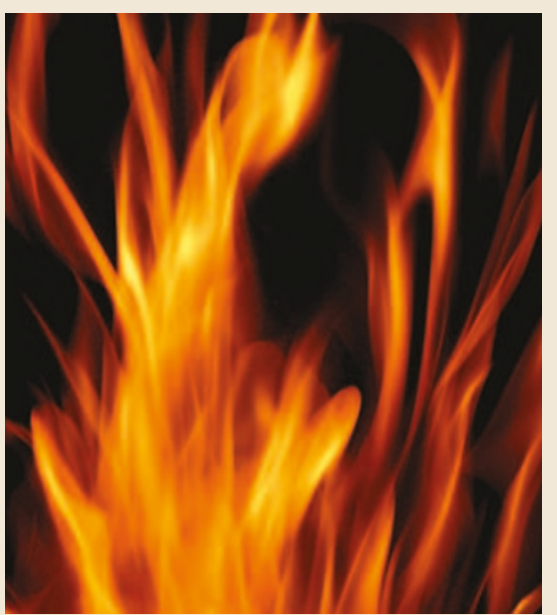

with several other cancers, further studies are needed to clarify the relationship between inflammation, transformation and the role of NK cells.

Jenny Bangham, Assistant Editor, Nature Reviews Cancer

(1) References and links ORIGINAL RESEARCH PAPER Carrington, M. et al. Hierarchy of resistance to cervical neoplasia mediated by combinations of killer immunoglobulin-like receptor and human leukocyte antigen loci. J. Exp. Med. 201, 1069-1075 (2005) 